\title{
The Research of Urban Rail Transit Integrated Automation System Based on Cloud Computing
}

\author{
Wu Qin ${ }^{1, a}$ \\ ${ }^{1}$ TianJin Railway Technical and Vocational College, China \\ azhaoqinwo@sina.com
}

\begin{abstract}
Keywords: Urban rail transportation, Integrated automation systems, Integrated information management system
\end{abstract}

\begin{abstract}
This paper analyzes the state of the art of urban rail transit integrated automation system, combining automation, information technology, intelligent trend in industrial applications the depth of integration, noting that urban rail transit integrated automation system will be based on existing real-time automation for the main purpose of the application by introducing multi-core parallel processing, server clustering, mobile applications and other mainstream information technology, will have the ability to handle huge amounts of data, users can meet the growing requirements of information integration and information security requirements, support and lead to the intelligent system integrated automation system direction.
\end{abstract}

\section{Introduction}

Urban Rail Transit integrated automation system (ISCS) refers to the urban rail transit lines in all electrical and electro-mechanical equipment for automated hierarchical distributed computer integrated systems, including internal integrated subsystems, and with the other Professional automation system interconnection, information sharing, promote high-efficiency operation of urban rail transit [1]. As a general SCADA system in urban rail transit industry specific applications, integrated automation system with a systematic approach to decentralized automation systems link into an organic whole, to achieve orbit information exchange between the various professional transportation systems, resource sharing, improve the coordination capacity of the system to efficiently realize the linkage between systems, improve the overall level of rail traffic automation, enhanced to deal with various emergency response capabilities, improve rail traffic operation and management level [1], to improve the rail service quality and service levels, and better for the majority of passenger service, lay the foundation for the construction of digital rail transportation, improve rail transport in favor of resource management, improve economic efficiency.

\section{Rail Integrated Automation System Design Principles}

Rail automation system design should follow the following design principles:

Advance. Planning and design of the overall system solution, each subsystem design should reflect the advanced nature of the principle, from the grand vision on the overall planning, a moderate advance in technology, the use of equipment, products and software not only mature but also representative of the world of technology level, in line with the mainstream of technology development.

Security, reliability. Rail automation system is rail transportation safety, efficiency and an important guarantee for efficient operation and management, secure and reliable system is critical, the system should be designed to give priority to mature technology and products [3].

Openness. Rail automation system is an open system, through deep integration, interconnection and integration can be achieved with integrated manner, by means of trusteeship and control integration platform for the integrated management of each subsystem should have standard protocols and interfaces of each subsystem can integrate different brands, different art systems and products. 
Scalability. Rail automation system design should fully take into account the size of the system expansion needs, system functions, system performance, a modular architecture.

Economy, practicality. Subway operators should ensure efficiency, energy conservation, safety and reliability of height, system planning and design, in order to improve operational efficiency, to save energy, resources and manpower as the goal, give full consideration to the practical and benefit systems, and strive to get the maximum return on investment [5].

Serviceability. System should be standardized, modular, structured technical and product support online maintenance. Integrated automation system to achieve a module and function routine universal SCADA system, as shown in Figure 1. The main components include distributed communication software bus, scripts, secondary development tools, interfaces and acquisition, real-time database, alarms, logs, historical data, reports, command, authority, security management, remote management. Inter-module communication with each other through a distributed communication software bus, and implement FEP, servers and workstations by different combinations of functional requirements of the deployment of the module [2].

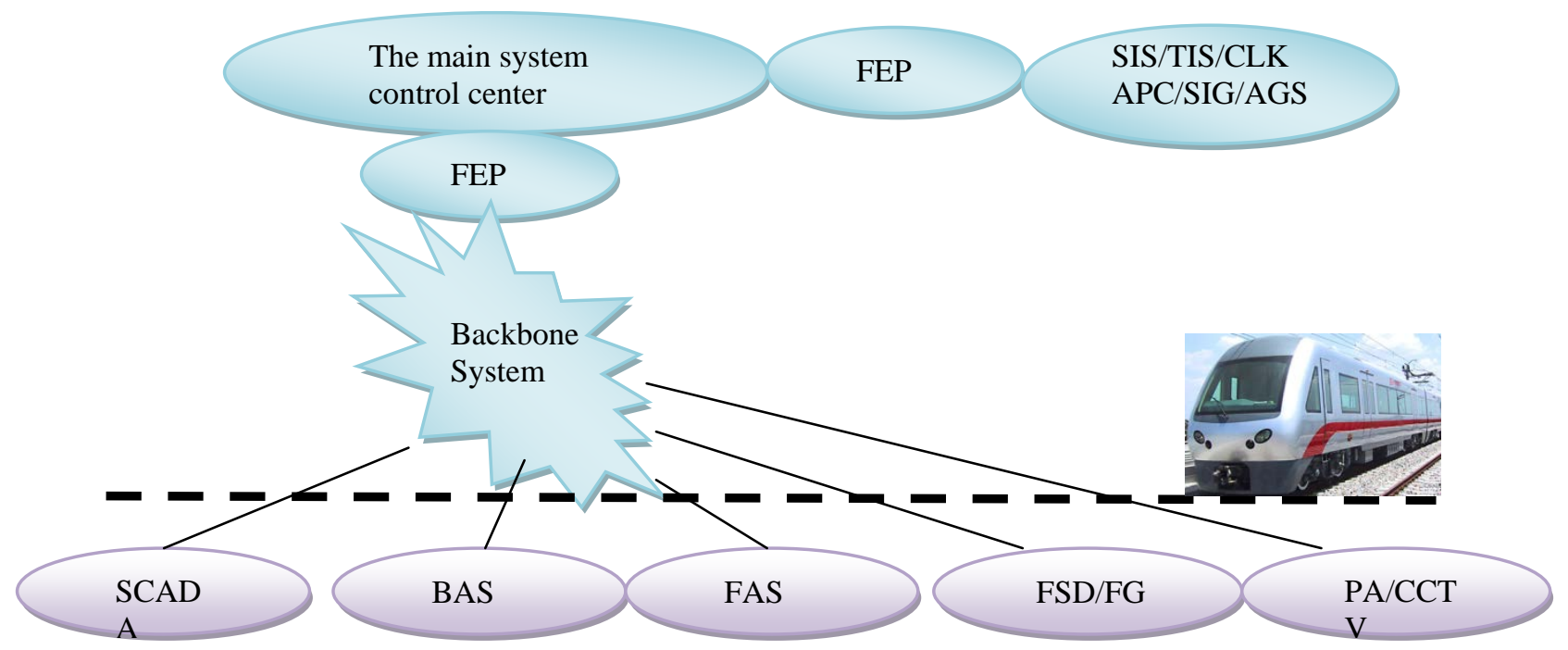

Fig.1 Integrated Automation System

\section{Techniques of Integrated Automation System}

As a manufacturer of computer-based process control and SCADA systems, integrated automation systems can run on-site monitoring and control equipment to achieve the various functions of data acquisition, device control, measurement, parameter adjustment and a variety of signal alarm. Integrated automation system has complete information, improve efficiency and proper understanding of system operation [3], accelerate decision-making, can help quickly diagnose system faults status and other advantages, now has become an indispensable tool for automated scheduling.

An important concept of integrated automation system in the development process should be to provide users with easy-to-use system extensions and: start small, but whether from short-term and long-term perspective, can easily be online according to the user's needs expansion. This design concept to ensure the system does not prematurely lose value [3]. However, with the development of technology, management and control integration and "information technology and industrialization, the depth of integration," as well as management of increasingly sophisticated, real-time automation of existing main application purpose integrated automation system can not fully meet the subway safe and efficient operation and management of needs, especially system software architecture in scheduling, production planning and workflow processing deficiencies that exist in the system has been seriously restricted from the process control level to the manufacturing execution level and the expansion of operating management, the impact of a comprehensive integrated automation system from the intelligent the development of information management systems. To solve these problems, 
integrated automation system requires an existing real-time automation based on the combination of the rapid development of information technology, through the introduction of a service-oriented architectures, multi-core parallel processing, parallel extensions mature IT server clusters, mobile applications, security systems, etc. technology, build a new generation of intelligent integrated information management system to meet the growing domestic and international users information integration requirements, providing a good user experience.

Integration and Information Systems. Most of the existing integrated automation system with the upper production scheduling and planning, quality management, equipment management, security management, office automation and other management information systems are separate, or only a simple way for simple data transmission from the bottom up, you cannot with the upper information systems integrated into an organic unity. In accordance with the requirements of the development of new industrial and enterprise information technology, automation management and control should be set in one, it contains low-level automation control and high-level management. Enterprise information system for automation of a higher requirement, it contains from business management, manufacturing execution level, the process control layer until the whole process field device level, covering the entire system from sensor starts to optimize the operation of all low-level control and senior management [4]. To ensure that the entire control process does not precipitate all useful information and loss, to facilitate real-time coordination and strengthen support for the top decision-making aid, we should establish the concept of a global, unified information platform, to overcome the "islands of automation", "islands of information" phenomenon, to achieve seamless integration of management and control integration.

The entire system should be hierarchical distributed system architecture, software systems should be modular structure, built as an open, scalable systems to facilitate flexible configuration system, extensions and performance improvements, support for sustainable enterprise business process reengineering, adaptation renovation and upgrading of enterprises.

Security system. Security is not because of man, machine and the interaction between the media and lead to system damage, personal injury, or loss affected task time [5]. At present, there is no integrated automation systems mostly conducted in accordance with the design and construction of safety-related systems, the lack of system security analysis, hazard identification and safety management. With the further development of integrated automation systems, security automation system integrated more and more attention and concern, the security problem is not resolved will also restrict the development and promotion of integrated automation systems.

IEC61508 international standards of the various stages of electrical / electronic / programmable electronic safety-related systems software and hardware life cycle tasks and objectives, requirements specification to provide a safe way to develop is to carry out rail safety assessment and demonstrate an important reference standard [5]. In the rail transport sector, Europe to IEC61508 standards, absorb the essence of the standards, the development of the railway industry standards, which includes the following four parts:

EN-50126 Railway applications: Reliability, Availability, Maintainability and Safety (RAMS) specification and description.

EN-50128 Railway applications: railway control and protection systems software.

EN-50129 Railway applications: safety-related electronic systems.

EN-50159 Railway applications: communications, signaling and processing systems.

Integrated Automation System should refer to these standards for the design, development, manufacture, operation and maintenance, making the system reaches safety integrity level SIL2 / SIL3.

A new generation of urban rail transit integrated information management system. To meet the technological development of urban rail transit integrated automation system, Beijing and advantage the company as the leading systems integrators and proprietary integrated automation system software provider, we presented a new generation of urban rail transit integrated information management system MACS-I2SCS the system framework, the establishment of information integration from the architecture unified architecture, integrated SCADA and management systems 
from a technical, as shown below, and is performed on a typical application design integrated automation system project Shenzhen-Hong Kong MTR wide [6].

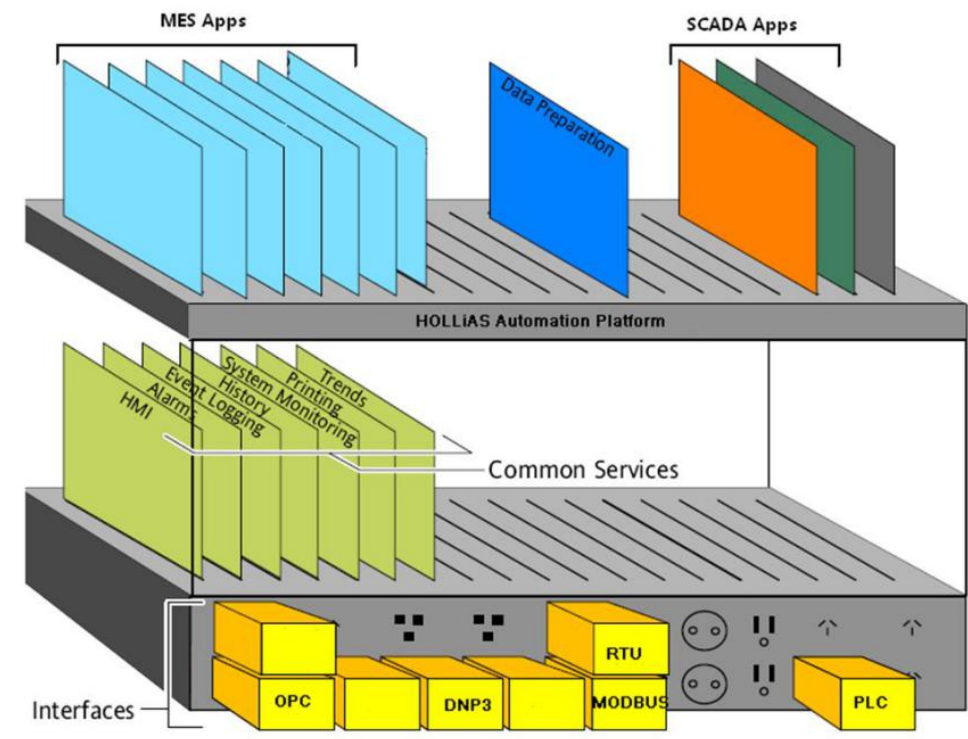

Fig.2 The new generation of urban rail transit integrated information management system unified architecture

The system platform technology roadmap includes:

$>$ To serve as the center of the SOA system architecture, through SOA connectors to third-party systems can easily integrate or present system. SOA connector is a Web service using WSDL description of the interface, providing developers SOA connector framework and interface specifications for third-party system or the system now.

$>$ To provide data services based on distributed server clusters, building object-oriented real-time database, using magent for server clusters extended to support Oracle and MySQL database with support for UNIX, LINUX and WINDOWS operating system.

$>$ Based on ESB service interface framework, messaging middleware using ActiveMQ.

$>$ The HTML5-based 2D / 3D user interface, the application of high real-time requirements to achieve plug-ins.

$>$ using TETRA radio communications technology public and private network access, provides automated handheld mobile terminal based tablet PC, smartphone or mini-panel.

> Press SIL2 standard safety-related systems development. EN50126 / 128/129 of SIL2 on safety-related systems and software development and approval of the respective responsibilities, procedures, documentation and put forward specific requirements.

In the new generation of urban rail transit integrated information management system MACS-I2SCS, the establishment of automation and information integration framework and interface specifications, easy to integrate existing software assets, improve software reusability, reduce costs, and also for the future expansion leaving a space. By means of MACS-I2SCS system platform, enabling management and control integration, can be significantly expanded in the following areas

Mobile Intelligent Systems. Currently the private network and public network wireless communication technology is very mature and widely used types of automation and information systems [7]. In the urban rail transit industry applications, based on the TETRA (Terrestrial Trunked Radio, namely land Trunked Radio) digital trunking communication technology has been widely used in train operations management and control systems. By combining existing integrated automation system and a mobile communication system that provides for the daily operation and management traffic urban rail transportation, power, and environmental control of local and remote scheduling service, and mobile service scheduling, traffic analysis, energy analysis, wireless emergency command service. Integrated automation systems via deep integration of wireless communication systems, to achieve comprehensive automation system existing movable and remote service capabilities, thus changing the shortcomings in the past and used the system can only operate 
at the station and the central control room fixed terminals, mobile value-added play unique advantages for subway maintenance personnel to provide mobile service function, providing management contingency plans for emergency situations such as fire subway, command and decision support capabilities for Metro dispatchers and managers to provide mobile-based tablet PCs, smart phones or miniature panel automation terminals, mobile office, is conducive to enhancing the intelligence level of the system [7].

\section{Conclusion}

Existing urban rail transit automation systems, real-time automation applications as the main purpose of achieving power dispatching, electrical and mechanical equipment automation, station operating status monitoring, and other functions, in terms of architecture and interface design focused primarily on real-time automation and data processing equipment, architectural deficiencies exist in production scheduling, production planning and workflow processing. On the other hand, due to the rapid development of IT technology among the existing system does not achieve service-oriented architecture (SOA), multi-core parallel processing, server clustering, load balancing, parallel extensions, mobile applications and other technology has become mature, It can be introduced to the new generation of integrated information management system to meet the growing user information integration requirements. To build a new generation of integrated information management system integration of critical infrastructure, security and emergency command, multi-mode communications systems as a platform of urban rail transit integrated automation platform, scalable range of integrated automation systems and enhance the level of integrated automation systems, support and Leading integrated automation systems to intelligent direction, to achieve integrated management and control.

\section{References}

[1] Sh. Zhang and M. Wang, Study and discuss a number of issues of a new generation of integrated automation system, A modern urban rail transit, January 2010, pp.54-58.

[2] T.T. Wang and W.G. Wang, Modern urban rail transit integrated automation system design, Automation and Applications, 2012, pp,37-42.

[3] Q.L. Chou, Digital, information technology, automation Coal Outlook, A modern enterprise education, Tsinghua University Press, 2015, pp.74-78.

[4] B. Li, Based on the study and implementation of GIS Integrated Automation System, Detection and control, 2014, pp.22-26.

[5] W.X. Pu, Car trillion to build RAMS management of urban rail transit integrated automation system, Urban Rail Transportation Research, 2011, pp.11-18.

[6] Y.X. An, Traction power supply based on SOA architecture integrated SCADA systems, electrified railway, 2011, pp.31-36.

[7] X.P.Sun and L.L. Wang, Urban Rail Development Trend of Integrated Automation Technology, Beijing: Mechanical Industry Press, 2011, pp.45-47. 